\title{
Vibrational Study and Force Field of the Citric Acid Dimer Based on the SQM Methodology
}

\author{
Laura Cecilia Bichara, ${ }^{1}$ Hernán Enrique Lanús, ${ }^{2}$ Evelina Gloria Ferrer, ${ }^{3}$ \\ Mónica Beatriz Gramajo, ${ }^{4}$ and Silvia Antonia Brandán ${ }^{1}$ \\ ${ }^{1}$ Cátedra de Química General, Instituto de Química Inorgánica, Facultad de Bioquímica, Química y Farmacia, \\ Universidad Nacional de Tucumán, Ayacucho 471, 4000 S. M. de Tucumán, Argentina \\ ${ }^{2}$ Cátedra de Fisicoquímica I, Instituto de Química Física, Facultad de Bioquímica, Química y Farmacia, \\ Universidad Nacional de Tucumán, San Lorenzo 456, T4000CAN S, M. de Tucumán., Argentina \\ ${ }^{3}$ Centro de Química Inorgánica, CEQUINOR/CCT, Departamento de Química, Facultad de Ciencias Exactas, \\ Universidad Nacional de La Plata, 47 y 115. CC. 962 (B1900AVV), 1900 La Plata, Argentina \\ ${ }^{4}$ Laboratorio de Fisicoquímica, Departamento de Física, Facultad de Ciencias Exactas y Tecnología, Universidad Nacional de Tucumán, \\ Avenida Independencia 1800, T4000CAN S. M. de Tucumán., Argentina \\ Correspondence should be addressed to Silvia Antonia Brandán, sbrandan@fbqf.unt.edu.ar
}

Received 11 February 2011; Accepted 14 April 2011

Academic Editor: Joel Bowman

Copyright ( $) 2011$ Laura Cecilia Bichara et al. This is an open access article distributed under the Creative Commons Attribution License, which permits unrestricted use, distribution, and reproduction in any medium, provided the original work is properly cited.

\begin{abstract}
We have carried out a structural and vibrational theoretical study for the citric acid dimer. The Density Functional Theory (DFT) method with the B3LYP/6-31G* and B3LYP/6-311++G** methods have been used to study its structure and vibrational properties. Then, in order to get a good assignment of the IR and Raman spectra in solid phase of dimer, the best fit possible between the calculated and recorded frequencies was carry out and the force fields were scaled using the Scaled Quantum Mechanic Force Field (SQMFF) methodology. An assignment of the observed spectral features is proposed. A band of medium intensity at $1242 \mathrm{~cm}^{-1}$ together with a group of weak bands, previously not assigned to the monomer, was in this case assigned to the dimer. Furthermore, the analysis of the Natural Bond Orbitals (NBOs) and the topological properties of electronic charge density by employing Bader's Atoms in Molecules theory (AIM) for the dimer were carried out to study the charge transference interactions of the compound.
\end{abstract}

\section{Introduction}

The structural and vibrational studies of citric acid are of chemical, biochemical, and technological interest [18]. The crystal and molecular structure of anhydrous citric acid was determined by Nordman et al. [6] and Glusker et al. [7] by means of X-ray crystal analyses. Both authors demonstrated that two molecules of the acid are linked through hydrogen bonds between their carboxylic groups originating the centre-symmetrical cyclic structure of the dimeric species. Many previous studies in relation to the chemical and spectroscopic properties of this acid as monomer have been reported [9-14] while an ab-initio/HF force field study for the vibrations of citric acid and citrate trianion was published by Tarakeshwar and Manogaran [15].
In the latter study, the calculated geometries and vibrational frequencies only for the monomer citric acid were used to assign the vibrational spectra. A complete vibrational characterization of this acid is very important to obtain information about chemical and physical components in food, biological materials, and pharmaceutical products at the molecular level $[14,16]$. So far, there are no publications about experimental or high-level theoretical studies on the geometries and force field for the dimer citric acid. Hence, obtaining reliable parameters by DFT theoretical methods is an appealing alternative to have a precise knowledge of the normal vibration modes. The aim of this paper is to report a DFT theoretical and experimental study of dimer citric acid by infrared spectroscopy in order to effect a complete assignment of every observed bands in the vibrational 
spectra by taking into account the presence of the dimeric species in the solid phase. For this purpose, the optimized geometries and frequencies for the normal vibration modes, considering the dimer with two units of the free acid, were calculated. For a complete and reliable assignment of the compound, the DFT calculations were combined with the SQMFF methodology [17-19] in order to fit the theoretical frequencies values to the experimental ones. In addition, the force constants were calculated and compared with those reported for the monomer by using the HF/4-21G method [15]. Here, we demonstrated that a DFT molecular force field for the dimer calculated by using the B3LYP/6$31 G^{*}$ combination is well represented. DFT normal mode assignments, in terms of the potential energy distribution, are in agreement with those obtained from the normal coordinate analysis. The total energy calculated with the 6$31 \mathrm{G}^{*}$ basis set for citric acid dimer was corrected for basis set superposition error (BSSE) by the standard Boys-Bernardi counterpoise method [20]. Besides, the dimer electronic properties were evaluated by NBO [21-24] and Atoms in Molecules (AIMs) $[25,26]$ studies in order to analyze the nature and magnitude of the intermolecular interactions.

\section{Experimental and Theoretical Calculations}

A pure anhydrous Mallinckrodt commercial sample of citric acid $\left(\mathrm{C}_{6} \mathrm{H}_{8} \mathrm{O}_{7}\right)$ (CA) was used. The FTIR spectrum of the solid substance in $\mathrm{KBr}$ pellets was recorded with a Bruker IFS 66 FTIR-spectrophotometer from 4000 to $400 \mathrm{~cm}^{-1}$. FTRaman spectrum was also collected on a Bruker IFS 66 FT-IR spectrophotometer provided with a NIR Raman attachment equipped with an Nd:YAG laser at $1064 \mathrm{~nm}$ laser.

The starting point for the dimer geometry optimization was a structure with bond lengths taken from the $\mathrm{X}$ ray crystal structure $[6,7]$. These initial geometries were optimized by using the hybrid B3LYP method $[27,28]$ with the $6-31 G^{*}$ and $6-311++G^{* *}$ basis sets, as implemented in the GAUSSIAN 03 program [29]. The atom structure and labelling can be seen in Figure 1. The electronic charge density topological analysis was performed by using the Atoms in Molecules (AIMs) methodology [25], by the AIM2000 program package [26] while the NBO calculations were performed by using the NBO 3.1 program [24], as implemented in the GAUSSIAN 03 package [29]. The harmonic wavenumbers and the valence force fields in Cartesian coordinates were calculated at the $\mathrm{B} 3 \mathrm{LYP} / 6-31 \mathrm{G}^{*}$ and 6$311++\mathrm{G}^{* *}$ levels of approximation. The resulting force fields were transformed into "natural" internal coordinates by using the MOLVIB program [30, 31]. The natural internal coordinates are presented in Table S1 of the Supplementary Material at doi/0.1155/2011/347072 and have been defined as proposed by Fogarasi et al. [32]. In this case, some coordinates are slightly different from those used in the previous study [15]. The six inter-monomer coordinates for this dimer are similar to those defined by other authors for other dimeric acids [33-36], which can be seen in Supplementary Table S2. Following the SQMFF procedure $[37,38]$ the harmonic force fields were evaluated at B3LYP/6$31 \mathrm{G}^{*}$ level. In the scaling procedure the diagonal force constants associated with a particular symmetry coordinate are multiplied by a small number of scale factors $\left(f_{i}, f_{j}\right.$, etc.). As a result, each off-diagonal constant is automatically scaled by the geometrical mean of factors $\left(f_{i} \times f_{j}\right) 1 / 2$ for the corresponding diagonal constants. Finally, the scale factors are adjusted by an iterative method until they reproduce the experimental wavenumbers as possible. In this case, we use the Rauhut and Pulay scale factors [17, 18]. The potential energy distribution (PEDs) components higher than or equal to $10 \%$ are subsequently calculated with the resulting SQM. The nature of every vibration modes was carried out by means of the GaussView program [39].

\section{Results and Discussion}

3.1. Geometries Optimization. Supplementary Table S3 shows the total energies and the corresponding dipole moment values for the dimer structures with the B3LYP method by using $6-31 \mathrm{G}^{*}$ and $6-311++\mathrm{G}^{* *}$ basis sets compared with the ones corresponding to two monomer units. The energy difference between the cyclic dimer and the two citric acid isolated monomers with the B3LYP/6-31G* method is $82.86 \mathrm{~kJ} \mathrm{~mol}^{-1}$ while by using the $6-311++\mathrm{G}^{* *}$ basis set the value at $68.64 \mathrm{kJmol}^{-1}$ decreases. Reva and Stepanian [40] predict an energy difference of $86.00 \mathrm{~kJ} \mathrm{~mol}^{-1}$ between the cyclic dimer and two isolated monomers of benzoic acid ( $a b$ initio/4-31G) while the calculated energy difference between their monomers is $40.12 \mathrm{~kJ} \mathrm{~mol}^{-1}$. In this case, the total energy for the citric acid dimer is smaller than the sum of the energies of each monomer (Supplementary Table S3) by using both basis sets. Such observation might mean that the dimer presence even in the gas phase is preferable to the isolated monomer, as observed in the 4-hydroxybenzoic acid dimer [36]. This result is very important to carry out the assignment of the vibrational spectra of this acid. In general, our theoretical values are in agreement with the experimental values for the citric acid $[6,7]$. The dimer stability was investigated by means of NBO [21-23] and AIM calculations [25]. In the NBO analysis, the second-order perturbation energies $\mathrm{E}^{(2)}$ (donor $\rightarrow$ acceptor) involving the most important delocalization for the dimer clearly reveal the energies and occupancies of the main contributions: $\mathrm{LP}(2) \mathrm{O} 19 \rightarrow \sigma^{*} \mathrm{O} 22-\mathrm{H} 21$ $\left(115.2 \mathrm{~kJ} \mathrm{~mol}^{-1}\right), \mathrm{LP}(2) \mathrm{O} 27 \rightarrow \sigma^{*} \mathrm{O} 19-\mathrm{H} 20\left(38.4 \mathrm{~kJ} \mathrm{~mol}^{-1}\right)$ and $\mathrm{LP}(2) \mathrm{O} 27 \rightarrow \sigma^{*} \mathrm{C} 3-\mathrm{H} 6 \quad\left(2.4 \mathrm{~kJ} \mathrm{~mol}^{-1}\right)$ to the delocalization energy due to the $\mathrm{OH}-\mathrm{O}$ bonds between both structures. In Bader's topological analysis [25] the localization of the Bond Critical Point (BCP) and Ring Critical Point (RCP) in the $\rho(r)$ and the Laplacian values $\nabla^{2} \rho(r)$ at these points is important to characterize molecular electronic structure. The critical point has the typical properties of the closed-shell interaction when the value of $\rho(r)$ is relatively low, the relationship $\left|\lambda_{1}\right| / \lambda_{3}$ is $<1$, and $\nabla^{2} \rho(r)$ is positive indicating that the interaction is dominated by the charge contraction away from the interatomic surface toward each nucleus. The topological properties for the citric acid dimer can be seen in Supplementary Table S4. The values obtained clearly reveal the formation of hydrogen bonds between two $\mathrm{COOH}$ 


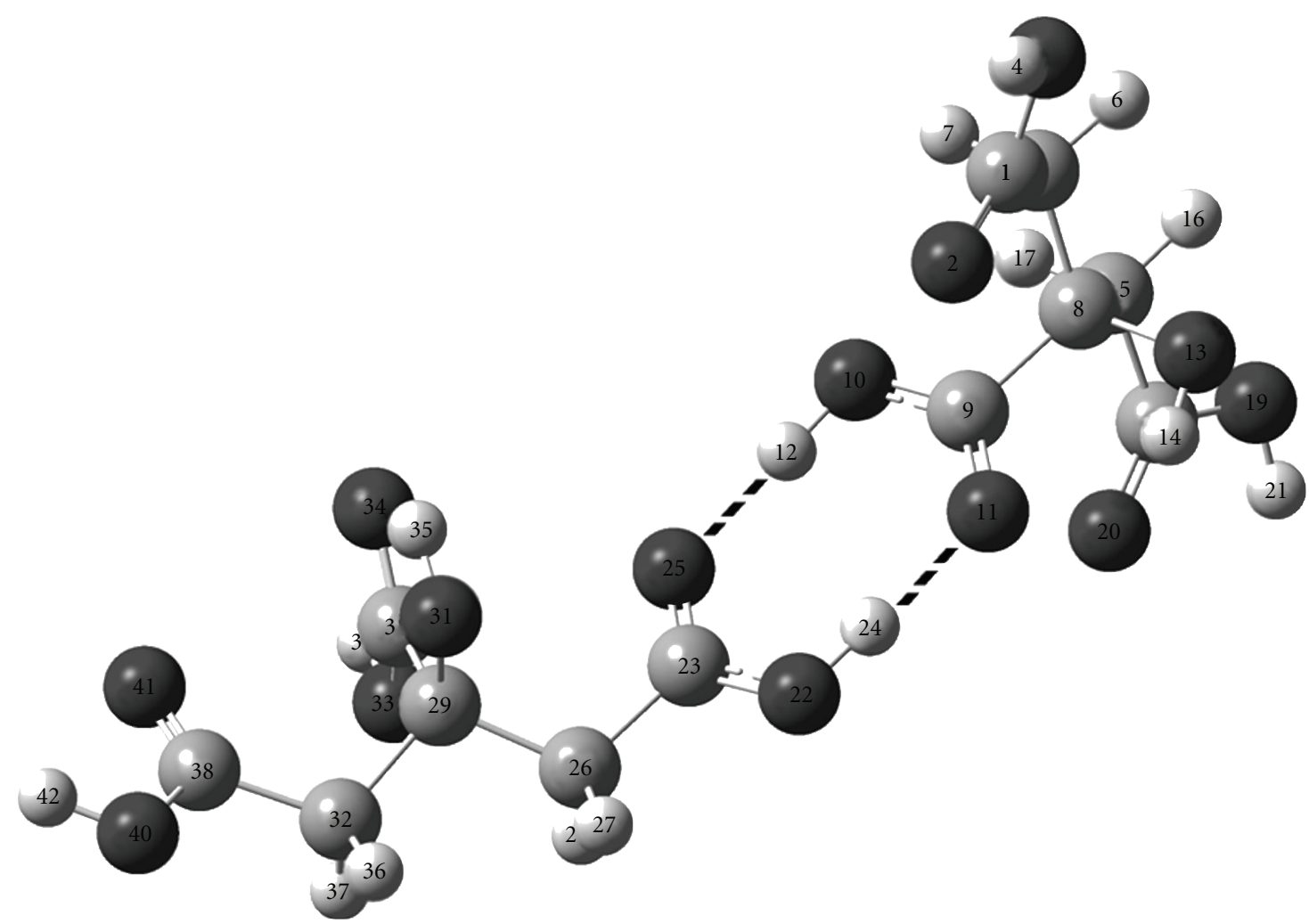

FIgURE 1: Theoretical structure of the citric acid dimer. The H-bonding is indicated by dashed lines.

groups of each monomer. In addition, the seven RCPs calculated by means AIM analysis for the dimer corroborate those contributions found in the NBO study.

\section{Vibrational Analysis}

The dimer's structure has $C_{1}$ symmetry and 120 vibrational normal modes where all vibrations are IR and Raman active. The recorded infrared spectra for the compound in the solid phase can be seen in Figure 2. The assignment of the experimental bands to the 120 expected normal vibration modes was made on the basis of the Potential Energy Distribution (PED) in terms of symmetry coordinates by using the B3LYP/6-31G* data and taking into account the previous assignment for the monomer [15] and for related molecules [33-36, 40]. Table 1 shows the observed frequencies and the assignment of the vibrational normal modes while the PED based on the 6-31G* basis set is shown in Supplementary Table S5. The theoretical calculations reproduce the normal wavenumbers by using the $6-31 \mathrm{G}^{*}$ basis set, with an initial rmsd value of $11.5 \mathrm{~cm}^{-1}$ while when the SQMFF method is applied by using Pulay's scaling factors, the final value is $1.5 \mathrm{~cm}^{-1}$. The best results are obtained with a B3LYP/6$31 G^{*}$ calculation because the used transferable scale factors are only defined for this method. The SQM force fields for the compound dimer can be obtained at request. The broad band observed between 3400 and $2200 \mathrm{~cm}^{-1}$ in the experimental IR spectra of the compound in the solid phase can be attributed to the $\mathrm{O}-\mathrm{H}$ hydrogen bond formed by the spatial arrangement of molecules in the lattice crystal
$[41,42]$. Note that the theoretical infrared spectra for the monomer structure demonstrate a little agreement with the experimental spectrum (Figure 2), especially in the high wavenumbers $\mathrm{cm}^{-1}$ region. On the other hand, in the theoretical Raman spectrum shown in Figure 3, in relation to the experimental one, a higher intensity in the bands attributed to the $\mathrm{OH}$ stretching is observed. The existence of molecules in the solid phase as dimer justifies such a difference because this species restricts the motion of the $\mathrm{COOH}$ groups and is strongly affected by the double hydrogen bond, as was also observed in the 4-hydroxybenzoic acid in solid state [36]. Thus, the wavenumbers in the dimer are shifted with respect to the monomer and the splitting of the antisymmetric and symmetric modes is particularly large. In this compound, both monomer and dimer structures are present in the solid phase and, for this reason, a comparison between the average calculated infrared spectra from B3LYP/6-31G* level for the monomer and dimer by using average wavenumbers and intensities with the corresponding experimental ones demonstrates a good correlation, especially in the high wavenumbers region. The discussion of the assignment of the most important groups for the compound (see Table 1 and Supplementary Table S5) is presented as follows.

\subsection{Band Assignments}

$\mathrm{OH}$ Modes. In the previous assignment for the monomer the theoretical HF/4-21G calculations [15] predict that the behaviour of the central $\mathrm{COOH}$ group is different from the terminal groups, as in our case by using the B3LYP/6-31G* 
TABLE 1: Observed and calculated wavenumbers $\left(\mathrm{cm}^{-1}\right)$ and assignments for citric acid dimmer.

\begin{tabular}{|c|c|c|c|c|c|c|}
\hline \multicolumn{2}{|c|}{ Experimental $^{\mathrm{a}}$} & \multicolumn{3}{|c|}{ Monomer $^{\mathrm{b}}$} & \multicolumn{2}{|c|}{ Dimer $^{\mathrm{a}}$} \\
\hline IR & Raman & IR & Raman & Assignment & SQM $^{\mathrm{c}}$ & Assignment $^{\mathrm{a}}$ \\
\hline & $3535 \mathrm{vw}$ & & & $v \mathrm{O}-\mathrm{H}$ & 3537 & $v \mathrm{O} 33-\mathrm{H} 39$ \\
\hline & $3535 \mathrm{vw}$ & & & $v \mathrm{O}-\mathrm{H}$ & 3527 & $v \mathrm{O} 3-\mathrm{H} 4$ \\
\hline \multirow[t]{2}{*}{$3498 \mathrm{~s}$} & & & & $v \mathrm{O}-\mathrm{H}$ & 3527 & $v \mathrm{O} 19-\mathrm{H} 21$ \\
\hline & $3480 \mathrm{vw}$ & & & $v \mathrm{O}-\mathrm{H}$ & 3525 & $v \mathrm{O} 40-\mathrm{H} 42$ \\
\hline $3446 \mathrm{~m}$ & 3446 vw & & & & 3482 & $v \mathrm{O} 13-\mathrm{H} 14$ \\
\hline $3350 \mathrm{sh}$ & $3342 \mathrm{vw}$ & & & & 3470 & $v \mathrm{O} 31-\mathrm{H} 35$ \\
\hline $3291 \mathrm{~s}$ & $3276 \mathrm{vw}$ & & & & 3066 & $\mathrm{O} 22-\mathrm{H} 24$ \\
\hline \multicolumn{7}{|l|}{3247 sh } \\
\hline \multicolumn{7}{|l|}{$3040 \mathrm{sh}$} \\
\hline $3035 \mathrm{sh}$ & & & & & 3012 & va $\mathrm{CH}_{2}$ op \\
\hline 2994 w & $2996 \mathrm{w}$ & & 3000 & va $\mathrm{CH}_{2}$ & 3009 & va $\mathrm{CH}_{2}$ op \\
\hline 2994 w & $2996 \mathrm{w}$ & & & & 3006 & va $\mathrm{CH}_{2}$ ip \\
\hline \multirow[t]{4}{*}{2994 w } & $2996 \mathrm{w}$ & & 2975 & va $\mathrm{CH}_{2}$ & 3003 & va $\mathrm{CH}_{2}$ ip \\
\hline & $2982 \mathrm{~m}$ & & & & 2952 & vs $\mathrm{CH}_{2}$ ip \\
\hline & $2982 \mathrm{~m}$ & & 2950 & vs $\mathrm{CH}_{2}$ & 2951 & vs $\mathrm{CH}_{2}$ ip \\
\hline & $2982 \mathrm{~m}$ & & $2934(21)$ & vs $\mathrm{CH}_{2}$ & 2946 & vs $\mathrm{CH}_{2}$ op \\
\hline $2950 \mathrm{w}$ & $2961 \mathrm{w}$ & & & & 2945 & vs $\mathrm{CH}_{2}$ op \\
\hline $2933 \mathrm{w}$ & $2929 \mathrm{~m}$ & & & & 2942 & O10-H12 \\
\hline 1756 vs & & & & & 1786 & vs $\mathrm{C}=\mathrm{O}_{1}$ \\
\hline \multirow[t]{3}{*}{$1745 \mathrm{sh}$} & $1750 \mathrm{w}$ & & & & 1783 & $\nu_{\mathrm{s}} \mathrm{C}=\mathrm{O}_{2}$ \\
\hline & $1735 \mathrm{~s}$ & & & & 1775 & va $\mathrm{C}=\mathrm{O}_{2}$ \\
\hline & $1735 \mathrm{~s}$ & & & & 1771 & va $\mathrm{C}=\mathrm{O}_{1}$ \\
\hline 1708 vs & $1708 \mathrm{w}$ & $1706 s$ & $1736(10)$ & $\nu \mathrm{C}=\mathrm{O}$ & 1726 & va $\mathrm{C}=\mathrm{O}_{3}$ \\
\hline \multirow[t]{3}{*}{$1698 \mathrm{sh}$} & 1693 vs & $1695 \mathrm{sh}$ & $1693(11)$ & $\nu \mathrm{C}=\mathrm{O}$ & 1677 & vs $\mathrm{C}=\mathrm{O}_{3}$ \\
\hline & 1493 vs & & & & 1483 & $\delta \mathrm{s} \mathrm{COH}$ \\
\hline & $1469 \mathrm{w}$ & & & & 1463 & va C- $-\mathrm{C}_{2}$ \\
\hline $1430 \mathrm{~m}$ & $1432 \mathrm{~m}, \mathrm{br}$ & $1427 \mathrm{~s}$ & $1425 \mathrm{~m}$ & $\begin{array}{l}\delta \mathrm{CH}_{2} \\
\delta \mathrm{CH}_{3}\end{array}$ & 1447 & $v$ C29-C30 \\
\hline \multirow[t]{2}{*}{$1430 \mathrm{~m}$} & $1432 \mathrm{~m}, \mathrm{br}$ & & & & 1430 & $\delta \mathrm{CH}_{2}$ ip \\
\hline & $1424 \mathrm{sh}$ & & & & 1426 & $\delta \mathrm{CH}_{2}$ ip \\
\hline \multirow[t]{2}{*}{$1420 \mathrm{sh}$} & & & & & 1417 & $\delta \mathrm{CH}_{2}$ op \\
\hline & $1413 \mathrm{w}$ & & & & 1412 & $\delta \mathrm{CH}_{2}$ op \\
\hline $1389 \mathrm{~m}$ & $1391 \mathrm{~s}$ & $1389 \mathrm{~s}$ & $1389(9)$ & $\begin{array}{c}\delta \mathrm{COH}, \\
\text { wag } \mathrm{CH}_{3}\end{array}$ & 1402 & $\delta \mathrm{a} \mathrm{COH}$ \\
\hline $1389 \mathrm{~m}$ & & & & & 1385 & wag $\mathrm{CH}_{2}$ op \\
\hline \multirow[t]{2}{*}{$1389 \mathrm{~m}$} & & & & & 1383 & $\delta \mathrm{C} 8-\mathrm{O} 13-\mathrm{H} 14$ \\
\hline & $1365 \mathrm{w}$ & & & & 1381 & wag $\mathrm{CH}_{2}$ ip \\
\hline $1358 \mathrm{w}$ & & $1350 \mathrm{w}$ & $1350 \mathrm{w}$ & $\tau \mathrm{CH}_{2}$ & 1352 & $\rho \mathrm{CH}_{2}$ ip \\
\hline $1340 \mathrm{sh}$ & $1347 \mathrm{w}$ & $1325 \mathrm{vw}$ & & wag $\mathrm{CH}_{2}$ & 1334 & $\delta \mathrm{s} \mathrm{COH}$ \\
\hline $1308 \mathrm{w}$ & & & & & 1308 & $\delta \mathrm{s} \mathrm{COH}$ \\
\hline $1308 \mathrm{w}$ & & & & & 1306 & $\begin{array}{c}\text { wag } \mathrm{CH}_{2} \text { ip, } \\
\delta \mathrm{C} 38-\mathrm{O} 40-\mathrm{H} 42\end{array}$ \\
\hline $1308 \mathrm{w}$ & $1301 \mathrm{vw}$ & & & & 1303 & $\delta \mathrm{a} \mathrm{COH}$ \\
\hline \multirow[t]{3}{*}{$1292 \mathrm{w}$} & $1291 \mathrm{vw}$ & & & & 1303 & $\rho \mathrm{CH}_{2}$ ip, wag $\mathrm{CH}_{2}$ ip \\
\hline & $1285 \mathrm{vw}$ & $1280 \mathrm{w}$ & & $\begin{array}{c}\delta \mathrm{COH}, \\
\text { wag } \mathrm{CH}_{3}\end{array}$ & 1289 & $\rho \mathrm{CH}_{2}$ op \\
\hline & $1256 \mathrm{vw}$ & & & & 1266 & va C-O \\
\hline $1242 \mathrm{~m}$ & & & & & 1258 & vs $\mathrm{C}-\mathrm{O}$ \\
\hline
\end{tabular}


Table 1: Continued.

\begin{tabular}{|c|c|c|c|c|c|c|}
\hline \multicolumn{2}{|c|}{ Experimental $^{\mathrm{a}}$} & \multicolumn{3}{|c|}{ Monomer ${ }^{b}$} & \multicolumn{2}{|r|}{ Dimer $^{\mathrm{a}}$} \\
\hline IR & Raman & IR & Raman & Assignment & $\mathrm{SQM}^{\mathrm{c}}$ & Assignment $^{\mathrm{a}}$ \\
\hline $1214 \mathrm{~m}$ & $1229 \mathrm{w}$ & & & & 1219 & $\rho \mathrm{CH}_{2}$ ip \\
\hline $1214 \mathrm{~m}$ & $1211 \mathrm{~m}$ & $1210 \mathrm{w}$ & $1205 s$ & $\tau \mathrm{CH}_{2}$ & 1212 & $\rho \mathrm{CH}_{2}$ op \\
\hline $1199 \mathrm{sh}$ & $1201 \mathrm{w}$ & $1195 \mathrm{w}$ & & $v \mathrm{C}-\mathrm{O}$ & & \\
\hline \multirow[t]{2}{*}{$1174 \mathrm{~s}$} & $1179 \mathrm{w}$ & $1175 \mathrm{~s}$ & & $\delta \mathrm{COH}$ & 1169 & $v \mathrm{C} 30-\mathrm{O} 33$ \\
\hline & $1167 \mathrm{w}$ & & & & 1155 & $\nu \mathrm{C} 18-\mathrm{O} 19$ \\
\hline $1140 \mathrm{~s}$ & & $1141 \mathrm{~s}$ & $1141(3)$ & $v \mathrm{C}-\mathrm{O}$ & 1145 & $v \mathrm{C} 38-\mathrm{O} 40$ \\
\hline $1140 \mathrm{~s}$ & $1140 \mathrm{w}$ & & & & 1140 & $v \mathrm{C} 1-\mathrm{O} 3$ \\
\hline $1140 \mathrm{~s}$ & $1130 \mathrm{w}$ & & & & 1127 & $v \mathrm{C} 8-\mathrm{O} 13$ \\
\hline \multirow[t]{2}{*}{$1081 \mathrm{w}$} & $1083 \mathrm{~m}$ & $1090 \mathrm{w}$ & $1080 \mathrm{~s}$ & $v \mathrm{C}-\mathrm{C}$ & 1112 & $\delta \mathrm{a} \mathrm{COH}$ \\
\hline & $1053 \mathrm{~m}$ & $1053 \mathrm{~m}$ & $1053(6)$ & $\nu \mathrm{C}-\mathrm{C}$ & 1059 & $v \mathrm{C} 8-\mathrm{C} 5, v \mathrm{C} 8-\mathrm{C} 15$ \\
\hline \multirow[t]{4}{*}{$1051 \mathrm{w}$} & & & & & 1057 & $v \mathrm{C} 29-\mathrm{C} 32, v \mathrm{C} 29-\mathrm{C} 26$ \\
\hline & $1036 \mathrm{sh}$ & & & & 1039 & wag $\mathrm{CH}_{2}$ op \\
\hline & $1036 \mathrm{sh}$ & & & & 1036 & $\tau \mathrm{wCH}_{2}$ op \\
\hline & $966 \mathrm{vw}$ & & & & 926 & vs $\mathrm{C}-\mathrm{C}_{2}$ \\
\hline \multirow[t]{2}{*}{$945 \mathrm{w}$} & 943 vs & $943 \mathrm{~m}$ & $943(20)$ & $v \mathrm{C}-\mathrm{O}$ & 911 & vs $\mathrm{C}-\mathrm{C}_{1}$ \\
\hline & $914 \mathrm{sh}$ & & & & 886 & $\tau \mathrm{wCH}_{2}$ op \\
\hline $904 \mathrm{w}$ & $904 \mathrm{~m}$ & & & & 885 & $\tau \mathrm{wCH}_{2}$ op \\
\hline $904 \mathrm{w}$ & $904 \mathrm{~m}$ & & $900 \mathrm{~m}$ & $v \mathrm{C}-\mathrm{C}$ & 872 & $v$ C29-O31 \\
\hline \multirow[t]{7}{*}{$881 \mathrm{w}$} & $886 \mathrm{w}$ & & & & 871 & $\tau \mathrm{wCH}_{2}$ ip \\
\hline & $867 \mathrm{vw}$ & & & & 865 & $\begin{array}{l}\tau(\mathrm{O} 22-\mathrm{H} 24) \\
\tau(\mathrm{O} 10-\mathrm{H} 12)\end{array}$ \\
\hline & $850 \mathrm{vw}$ & & & & 857 & $v \mathrm{C} 32-\mathrm{C} 38$ \\
\hline & 842 vw & & & & 842 & va $\mathrm{C}-\mathrm{C}_{1}$ \\
\hline & $842 \mathrm{vw}$ & & & & 827 & $\gamma \mathrm{a}(\mathrm{OH}-\mathrm{O})^{\#}$ \\
\hline & 807 vw & & & & 800 & $\delta \mathrm{CCC}$ op \\
\hline & $795 \mathrm{sh}$ & $810 \mathrm{w}$ & & & 792 & $\gamma \mathrm{COO}_{2}$ \\
\hline $781 \mathrm{~s}$ & $784 \mathrm{vs}$ & & $786(29)$ & $v \mathrm{C}-\mathrm{C}$ & 783 & $\delta \mathrm{COO}_{2}$ \\
\hline \multirow[t]{2}{*}{$729 \mathrm{sh}$} & $723 \mathrm{w}$ & & & & 735 & $\delta \mathrm{COO}_{5}$ \\
\hline & $700 \mathrm{w}$ & & & & 685 & $\delta \mathrm{COO}_{4}$ \\
\hline \multirow[t]{3}{*}{$686 \mathrm{w}$} & & & $687(6)$ & $\gamma \mathrm{COO}$ & 674 & $\gamma \mathrm{COO}_{5}$ \\
\hline & $666 \mathrm{vw}$ & & & & 672 & $\gamma \mathrm{COO}_{3}$ \\
\hline & $656 \mathrm{sh}$ & $660 \mathrm{sh}$ & & & 655 & $\begin{array}{c}\gamma \mathrm{COO}_{2}, \tau(\mathrm{O} 3-\mathrm{H} 4) \\
\tau \mathrm{wCH}_{2} \text { op }\end{array}$ \\
\hline \multirow[t]{4}{*}{$640 \mathrm{w}$} & $642 \mathrm{w}$ & $645 \mathrm{w}$ & $645 \mathrm{w}$ & $\tau(\mathrm{OH})$ & 637 & $\tau(\mathrm{O} 40-\mathrm{H} 42)$ \\
\hline & $627 \mathrm{vw}$ & & & & 631 & $\tau(\mathrm{O} 3-\mathrm{H} 4)$ \\
\hline & $627 \mathrm{vw}$ & & & & 625 & $\tau(\mathrm{O} 19-\mathrm{H} 21)$ \\
\hline & $627 \mathrm{vw}$ & & & & 621 & $\delta \mathrm{COO}_{3}$ \\
\hline \multirow[t]{3}{*}{$599 \mathrm{~s}$} & $597 \mathrm{w}$ & $598 \mathrm{~s}$ & $598(5)$ & $\tau(\mathrm{OH})$ & 616 & $\begin{array}{c}\tau(\mathrm{O} 40-\mathrm{H} 42), \delta \mathrm{COO}_{5} \\
\delta \mathrm{C} 31-\mathrm{C} 29-\mathrm{C} 30, \rho \mathrm{COO}_{5}\end{array}$ \\
\hline & $574 \mathrm{w}$ & & & & 574 & $\delta \mathrm{COO}_{6}$ \\
\hline & $571 \mathrm{w}$ & & & & 566 & $\gamma \mathrm{COO}_{4}$ \\
\hline \multirow[t]{2}{*}{$550 \mathrm{w}$} & $559 \mathrm{~m}$ & & $556(7)$ & $\tau(\mathrm{OH})$ & 544 & $\rho \mathrm{COO}_{2}$ \\
\hline & $541 \mathrm{w}$ & & & & 537 & $\delta \mathrm{COO}_{1}$ \\
\hline \multirow[t]{2}{*}{$520 \mathrm{sh}$} & $528 \mathrm{vw}$ & & & & 519 & $\gamma \mathrm{COO}_{6}$ \\
\hline & $514 \mathrm{w}$ & & & & 499 & $\begin{array}{c}\gamma \mathrm{COO}_{3}, \tau \mathrm{wCH}_{2} \mathrm{ip} \\
\tau(\mathrm{O} 19-\mathrm{H} 21)\end{array}$ \\
\hline \multirow[t]{2}{*}{$504 \mathrm{w}$} & $509 \mathrm{sh}$ & $503 \mathrm{w}$ & & & 496 & $\gamma \mathrm{COO}_{1}$ \\
\hline & 492 vw & & & & 490 & $\tau(\mathrm{O} 33-\mathrm{H} 39)$ \\
\hline
\end{tabular}


TABle 1: Continued.

\begin{tabular}{|c|c|c|c|c|c|c|}
\hline \multicolumn{2}{|c|}{ Experimental $^{\mathrm{a}}$} & \multicolumn{3}{|c|}{ Monomer $^{\mathrm{b}}$} & \multicolumn{2}{|c|}{ Dimer $^{\mathrm{a}}$} \\
\hline IR & Raman & IR & Raman & Assignment & $\mathrm{SQM}^{\mathrm{c}}$ & Assignment $^{\mathrm{a}}$ \\
\hline $477 \mathrm{sh}$ & $476 \mathrm{vw}$ & & & & 485 & $\gamma \mathrm{COO}_{6}$ \\
\hline \multirow[t]{34}{*}{$438 \mathrm{vvw}$} & $453 \mathrm{w}$ & & & & 412 & $\rho \mathrm{COO}_{4}$ \\
\hline & $415 \mathrm{w}$ & $420 \mathrm{w}$ & & $\delta \mathrm{COO}$ & 398 & $\tau(\mathrm{O} 31-\mathrm{H} 35)$ \\
\hline & $397 w$ & $392 \mathrm{~s}$ & $383(11)$ & $\tau(\mathrm{OH})$ & 390 & $\delta \mathrm{C} 31-\mathrm{C} 29-\mathrm{C} 26$ \\
\hline & $382 \mathrm{~m}$ & & & & 387 & $\tau(\mathrm{O} 13-\mathrm{H} 14)$ \\
\hline & $367 \mathrm{w}$ & & & & 373 & $\rho \mathrm{COO}_{3}, \rho \mathrm{COO}_{1}$ \\
\hline & $367 \mathrm{w}$ & $358^{\mathrm{b}}$ & & $\delta \mathrm{COO}$ & 368 & $\rho \mathrm{COO}_{5}$ \\
\hline & $348 \mathrm{w}$ & $334^{\mathrm{b}}$ & & $\delta \mathrm{COO}$ & 345 & $\rho \mathrm{COO}_{6}$ \\
\hline & $323 \mathrm{vw}$ & $320^{\mathrm{b}}$ & & $\delta \mathrm{CCO}$ & 329 & $\delta \mathrm{C} 13-\mathrm{C} 8-\mathrm{C} 5$ \\
\hline & $306 \mathrm{w}$ & & & & 294 & 8C13-C8-C9 \\
\hline & $306 \mathrm{w}$ & & & & 271 & $\delta \mathrm{C} 26-\mathrm{C} 29-\mathrm{C} 32$ \\
\hline & $266 \mathrm{w}$ & & & & 251 & $\delta \mathrm{C} 31-\mathrm{C} 29-\mathrm{C} 30$ \\
\hline & $254 \mathrm{sh}$ & $255^{\mathrm{b}}$ & & $\rho \mathrm{CCC}$ & 236 & $\delta \mathrm{C} 5-\mathrm{C} 8-\mathrm{C} 15$ \\
\hline & $247 \mathrm{vw}$ & $245^{\mathrm{b}}$ & & $\delta \mathrm{CCC}$ & 222 & 8C9-C8-C5 \\
\hline & $237 \mathrm{vw}$ & $223^{\mathrm{b}}$ & & $\delta \mathrm{CCC}$ & 221 & $\delta \mathrm{C} 31-\mathrm{C} 29-\mathrm{C} 32$ \\
\hline & $217 \mathrm{w}$ & $187^{\mathrm{b}}$ & & $\delta \mathrm{CCC}$ & 180 & $\delta \mathrm{C} 26-\mathrm{C} 29-\mathrm{C} 30$ \\
\hline & $212 \mathrm{w}$ & & & & 174 & $\delta \mathrm{CCC}$ op \\
\hline & & $140^{\mathrm{b}}$ & & & 148 & $\nu \mathrm{s}(\mathrm{O}-\mathrm{H}-\mathrm{O})^{\#}$ \\
\hline & & & & & 127 & $\tau \mathrm{wCOO}_{3}$ \\
\hline & & & & & 122 & $\delta(\mathrm{OH}-\mathrm{O})^{\#}$ \\
\hline & & & & & 118 & $\tau \mathrm{wCOO}_{5}$ \\
\hline & & $105^{\mathrm{b}}$ & & $\delta \mathrm{CCC}$ & 108 & $v \mathrm{a}(\mathrm{O}-\mathrm{H}-\mathrm{O})^{\#}, \delta \mathrm{CCC}$ ip \\
\hline & & & & & 99 & $\tau \mathrm{wCOO}_{5}$ \\
\hline & & $88^{\mathrm{b}}$ & & $\tau \mathrm{wCOO}$ & 92 & $\delta \mathrm{CCC}$ ip \\
\hline & & $68^{\mathrm{b}}$ & & $\tau \mathrm{wCOO}$ & 72 & $\tau$ wCC ip \\
\hline & & & & & 57 & $\tau \mathrm{CC}_{2}$ \\
\hline & & & & & 53 & $\tau$ wop $^{\#}$ \\
\hline & & $44^{\mathrm{b}}$ & & $\tau \mathrm{wCOO}$ & 46 & $\tau \mathrm{wCC}$ op \\
\hline & & $39^{\mathrm{b}}$ & & $\tau \mathrm{wCOO}$ & 38 & $\tau \mathrm{wCOO}_{1}$ \\
\hline & & & & & 32 & $\tau \mathrm{wCOO}_{6}$ \\
\hline & & & & & 30 & $\tau \mathrm{wCOO}_{2}$ \\
\hline & & & & & 26 & $\begin{array}{c}\tau \mathrm{wCOO}_{1}, \tau \mathrm{wCOO}_{2} \\
\tau \mathrm{wCOO}_{3}\end{array}$ \\
\hline & & & & & 25 & $\tau \mathrm{CC}_{1}$ \\
\hline & & & & & 11 & $\gamma \mathrm{s}(\mathrm{OH}-\mathrm{O})^{\#}$ \\
\hline & & & & & 8 & $\tau \mathrm{wCOO}_{4}$ \\
\hline
\end{tabular}

$\bar{\nu}$ : stretching; $\delta$ : scissoring; wag: wagging; $\gamma$ : out-of plane deformation; $\rho$ : rocking; $\tau$ : torsion, $\tau$ W: twisting; a: antisymmetric; s: symmetric; s: strong; m: medium; w: weak; v: very; sh: shoulder; br: broad.

${ }^{\text {a }}$ This work.

${ }^{\mathrm{b}}$ Calculated by HF/4-21G method from [15].

'Theoretical values from SQM/B3LYP/6-31G* calculations for citric dimer acid.

\#Intermonomer coordinates.

level and, as a consequence, the central $\mathrm{OH}$ stretching mode belonging to that central group is calculated at higher wavenumbers than the other ones. In the dimer, those modes are assigned according to the SQM calculations; for this reason, the $\mathrm{OH}$ stretching modes are associated with the Raman band at $3535 \mathrm{~cm}^{-1}$, the IR bands at 3498, 3446, 3291 , and $2933 \mathrm{~cm}^{-1}$, and the shoulder located at $3350 \mathrm{~cm}^{-1}$.
The $\mathrm{C}-\mathrm{OH}$ scissoring mode belonging to the $\mathrm{COOH}$ groups involved in the $\mathrm{H}$-bonding formation is calculated at higher wavenumbers than the other ones (1483 and $1402 \mathrm{~cm}^{-1}$ ) probably because they are coupled with the corresponding $\mathrm{C}-\mathrm{O}$ stretching modes; for this reason, both modes are assigned to the bands at 1493 and $1389 \mathrm{~cm}^{-1}$. The remaining $\mathrm{C}-\mathrm{OH}$ scissoring modes are assigned to the shoulder and 


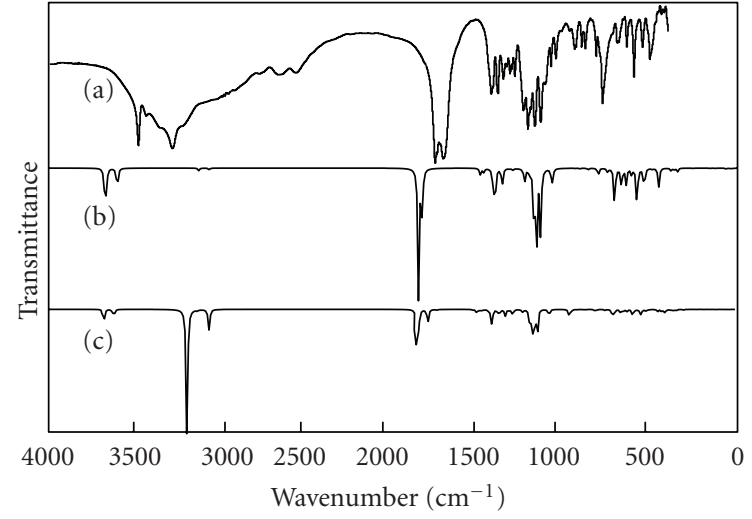

FIgURE 2: Comparison between (a) the experimental infrared spectrum in solid state, (b) the calculated infrared spectrum from B3LYP/6-31G* level for the monomer, and (c) the calculated infrared spectrum obtained from B3LYP/6-31G* level using average frequencies and intensities for both monomer and dimer species.

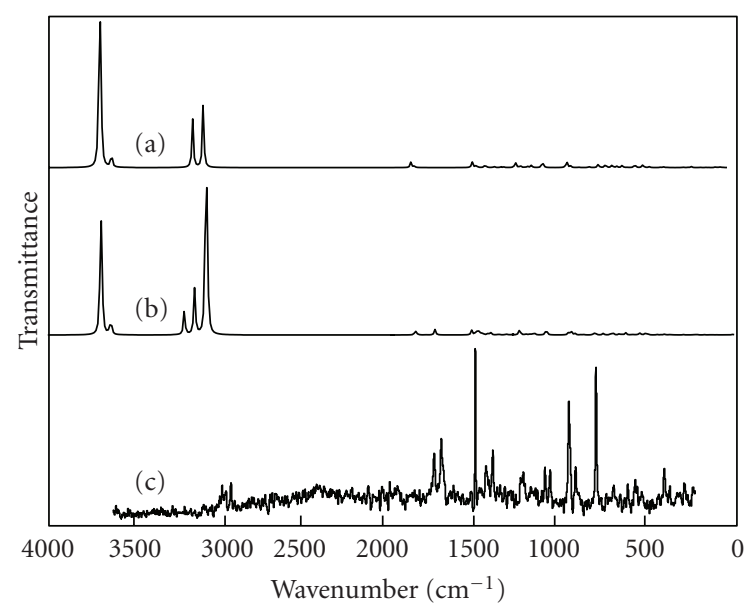

Figure 3: Comparison between (a) the calculated Raman spectrum from B3LYP/6-31G* level for the monomer, (b) the calculated Raman spectrum obtained from B3LYP/6-31G* level using average frequencies and intensities for both monomer and dimer species and (c) the experimental Raman spectrum in solid state.

IR bands at 1340,1308 , and $1081 \mathrm{~cm}^{-1}$, respectively, as seen in Table 1. In the region of lower wavenumbers, the $\mathrm{OH}$ torsion modes are expected and these modes are also affected by the presence of $\mathrm{H}$ bonding. Previously, those modes in the monomer were assigned, according to HF/4$21 \mathrm{G}$ calculations [15], to the bands at $645,598,556$, and $383 \mathrm{~cm}^{-1}$ while by using the B3LYP/6-31G* level they are calculated between 655 and $387 \mathrm{~cm}^{-1}$; for this reason, the $\mathrm{OH}$ torsion modes are associated with the Raman bands at 642, 627, 492 , and $415 \mathrm{~cm}^{-1}$.

$\mathrm{CH}_{2}$ Modes. These modes in the monomer were previously observed in the $3000-2900 \mathrm{~cm}^{-1}$ region [15]. In this case, the out-of-phase and in-phase antisymmetric stretching modes of these groups can be assigned to the shoulder and IR band
TABLE 2: Scaled force constants for the citric acid dimer.

\begin{tabular}{lcc}
\hline Description & $\begin{array}{c}\text { Dimer }^{\mathrm{a}} \\
\text { B3LYP/6-31G* }\end{array}$ & $\begin{array}{c}\text { Monomer }^{\mathrm{b}} \\
\text { HF/4-21G }\end{array}$ \\
\hline $\mathrm{f}(\nu \mathrm{O}-\mathrm{H})$ & 6.61 & 6.84 \\
$\mathrm{f}(\nu \mathrm{C}-\mathrm{C})$ & 3.92 & 4.34 \\
$\mathrm{f}(\nu \mathrm{C}=\mathrm{O})$ & 12.06 & 13.38 \\
$\mathrm{f}\left(\nu \mathrm{CH}_{2}\right)$ & 4.89 & 5.35 \\
$\mathrm{f}(\nu \mathrm{C}-\mathrm{O})$ & 6.07 & 6.11 \\
$\mathrm{f}\left(\delta \mathrm{CH}_{2}\right)$ & 0.75 & 0.55 \\
$\mathrm{f}\left(\right.$ wag $\left.\mathrm{CH}_{2}\right)$ & 1.15 & 0.65 \\
\hline
\end{tabular}

Units in mdyn $\AA^{-1}$ for stretching and mdyn $\AA$ rad $^{-2}$ for angle deformations. ${ }^{\mathrm{a}}$ This work.

${ }^{b}$ From [15].

at 3035 and $2994 \mathrm{~cm}^{-1}$, as indicated in Table 1, while the outof-phase and in-phase symmetric modes are assigned to the weak IR band at $2950 \mathrm{~cm}^{-1}$ and to the medium intensity Raman band at $2982 \mathrm{~cm}^{-1}$. In the monomer, the scissoring modes were assigned at $1427 \mathrm{~cm}^{-1}$ while for the dimer those modes were also clearly calculated in this region; thus, they are assigned to the bands at $1430,1424,1420$, and $1413 \mathrm{~cm}^{-1}$. In the above-mentioned study [15], the wagging modes were associated with the very weak IR band at $1325 \mathrm{~cm}^{-1}$. Here, as predicted by calculation, the bands at $1389,1365,1308$, 1292 , and $1036 \mathrm{~cm}^{-1}$ are assigned to the wagging modes. Previously, in the monomer the twisting and rocking modes were assigned at 1350 and $966 \mathrm{~cm}^{-1}$, respectively [15]. In the dimer, the IR bands located at 1358, 1292, and $1214 \mathrm{~cm}^{-1}$ and the Raman band at $1285 \mathrm{~cm}^{-1}$ are associated with the rocking modes, while the expected twisting modes are associated with the IR bands at 904 and $881 \mathrm{~cm}^{-1}$ and with the shoulder in the Raman spectrum at 1036, 656 and $514 \mathrm{~cm}^{-1}$.

COO Modes. In the previous studies for the monomer [15] the band at $1706 \mathrm{~cm}^{-1}$ and the shoulder at $1695 \mathrm{~cm}^{-1}$ were assigned to the $\mathrm{C}=\mathrm{O}$ stretching modes. Note that the band at $1706 \mathrm{~cm}^{-1}$ (Table 1) was related to the strong Raman band at $1735 \mathrm{~cm}^{-1}$ and assigned to the same mode in spite of the difference between them $\left(19 \mathrm{~cm}^{-1}\right)$. In the dimer, the six expected $\mathrm{C}=\mathrm{O}$ stretching modes are clearly predicted by SQM/B3LYP/6-31G* calculation at 1786, 1783, 1775, 1771, 1726 , and $1677 \mathrm{~cm}^{-1}$. On the other hand, the IR spectrum clearly shows the presence of more than one band in this region; thus, two very strong bands at 1756 and $1708 \mathrm{~cm}^{-1}$ can be seen, each band presenting a shoulder at 1745 and $1698 \mathrm{~cm}^{-1}$, respectively. Therefore, the IR and Raman bands between 1756 and $1698 \mathrm{~cm}^{-1}$ are clearly assigned to those modes. In this way, those shoulders should be also associated with other $\mathrm{C}=\mathrm{O}$ stretching modes, and so this fact would confirm the presence of the dimer in the solid state. In both HF/4-21G [15] and B3LYP/6-31G* calculations, the C$\mathrm{O}$ stretching modes are coupled with the $\mathrm{C}-\mathrm{OH}$ scissoring modes. In the monomer [15], those modes were assigned to the IR bands at 1195,1141 , and $1108 \mathrm{~cm}^{-1}$, while in the dimer, the IR bands at 1242,1174 , and $1140 \mathrm{~cm}^{-1}$ and the medium intensity Raman band at $904 \mathrm{~cm}^{-1}$ are assigned to these stretching modes. Previously, the medium intensity 
band in the IR spectrum at $1242 \mathrm{~cm}^{-1}$ was not observed and therefore not assigned. Taking into consideration their relative position, intensities, and the splitting predicted by calculation, the expected out-of-plane deformation modes $(\gamma \mathrm{COO})$, in-phase and out-of-phase modes, were predicted as pure modes between 792 and $485 \mathrm{~cm}^{-1}$ and coupled with other modes at 655 and $499 \mathrm{~cm}^{-1}$; for this reason, they were assigned to the bands at $807,795,686,574$, and $492 \mathrm{~cm}^{-1}$, as observed in Table 1. In the previous study [15], only one deformation mode $(\delta \mathrm{COO})$ was assigned to the band at $420 \mathrm{~cm}^{-1}$. Here, as predicted by calculation, those expected deformation modes for the dimer are assigned to the IR band and shoulder at 781 and $789 \mathrm{~cm}^{-1}$, respectively, and to the Raman bands at $700,627,574$, and $541 \mathrm{~cm}^{-1}$. The rocking modes $(\rho \mathrm{COO})$ for the monomer were not previously assigned and, in this case, the $\rho \mathrm{COO}$ modes were easily assigned to the IR bands at 599, 550, and $438 \mathrm{~cm}^{-1}$ and to the Raman bands at 367 and $348 \mathrm{~cm}^{-1}$. Finally, the twisting $(\tau \mathrm{wCOO})$ modes for the monomer were only predicted by the HF/4-21G level at $88,68,44$, and $39 \mathrm{~cm}^{-1}$ and, in our case, for the dimer they are predicted strongly coupled between them and in the low wavenumbers region; this way, they could not be assigned because there are not observed bands in the vibrational spectra in those regions.

Skeletal Modes. In the previous assignment [15], the bands in the infrared spectrum at 1090, 1053, 900, 850, and $786 \mathrm{~cm}^{-1}$ were attributed to the $\mathrm{C}-\mathrm{C}$ stretching modes, while the CCC deformation modes were not assigned because they appear at very low frequencies. Here, the bands in the IR spectrum at 1430, 1051 and $945 \mathrm{~cm}^{-1}$ and in the Raman spectrum at $1469,1053,966,850$, and $842 \mathrm{~cm}^{-1}$ are assigned to the $\mathrm{C}-\mathrm{C}$ stretching modes. The calculations predicted the CCC deformation modes in the lower wavenumbers region; for this reason, they are assigned to the Raman bands between 323 and $212 \mathrm{~cm}^{-1}$ while the two modes predicted at 108 and $92 \mathrm{~cm}^{-1}$ were not assigned.

Intermonomer Modes. The intermonomer vibrational modes of the dimer usually appear in the $270-10 \mathrm{~cm}^{-1}$ region [33$36,40]$ and they are related to restricted translations or rotations of one molecule against the other (see Supplementary Table S2). Here, the six intermonomer modes appear strongly coupled with other modes and they are observed in the Raman spectrum with the exception of the $\gamma_{a}(\mathrm{OH}-$ $\mathrm{O})$ mode that was predicted by calculations with a higher contribution PED $(42 \%)$ at $827 \mathrm{~cm}^{-1}$; for this reason, it is assigned to the very weak Raman band at $842 \mathrm{~cm}^{-1}$. Table 1 shows the theoretical assignments for the dimer intermonomer vibrational modes.

\section{Force Field}

The force constants for the dimer were estimated by using Pulay et al. $[37,38]$ scaling procedure as mentioned above. The force constants expressed in terms of simple valence internal coordinates were calculated from the corresponding scaled force fields by using the MOLVIB program [30, 31]. It is interesting to compare the principal force constants, which were collected in Table 2 and calculated at the B3LYP/631G* level for some vibrations, with those reported for the monomer of this acid by using the HF/4-21G method [15]. The calculated force constants values for the monomer by using the ab-initio method are slightly different from the values for the dimer by using the B3LYP/6-31G* level, as expected. The HF method overestimates the calculated force constants values due to the fact that it neglects the electron correlation and unharmony. On the other hand, the little variation in the $\mathrm{f}(\mathrm{C}=\mathrm{O})$ force constant value in the monomer with reference to the dimer is justified because in the latter species two molecules of the acid are linked by hydrogen bonds between their carboxylic groups; therefore, the involved $\mathrm{C}=\mathrm{O}$ bonds decrease the double bonds character. For this reason, a lower value in the $\mathrm{f}(\nu \mathrm{O}-$ $\mathrm{H}$ ) force constant can be also seen in the dimer in relation to the monomer. Obviously, the force constants related to the $\mathrm{CH}_{2}$ groups in the monomer have lower values than the dimer because in the latter species the number of those groups is higher.

\section{Conclusions}

We conclude the following.

(i) The assignment previously made for the monomer [15] was corrected and completed in accordance with the present theoretical B3LYP/6-31G* results. Also, the assignment of the intermonomer vibrational modes for the dimer is carried out and the assignments of the 120 normal vibration modes corresponding to citric acid dimer are reported.

(ii) Six intense bands in the infrared spectrum at 3498 , $3291,1756,1708,1174$, and $1140 \mathrm{~cm}^{-1}$ are reported to characterize the compound dimer.

(iii) Numerous bands of different intensities observed in the vibracional spectra not previously assigned, in this work were assigned to the citric acid dimer.

(iv) The NBO analysis reveals that in the citric acid dimer there are strong intramolecular interactions of charge transfer from $\mathrm{O}$ lone pairs to the $\sigma^{*}(\mathrm{O}-\mathrm{H})$ antibonds.

(v) The AIM studies of the charge density for the dimer satisfy the criteria of hydrogen bond interactions.

(vi) The complete B3LYP/6-31G* force field for the citric acid dimer was determined as well as the principal force constants for stretching and deformation modes.

\section{Acknowledgments}

This work was subsidized with grants from CIUNT (Consejo de Investigaciones, Universidad Nacional de Tucumán) and CONICET (Consejo Nacional de Investigaciones Científicas y Técnicas, R. Argentina). The authors thank Professor Tom Sundius for his permission to use MOLVIB. 


\section{References}

[1] H. Eggerer, U. Remberger, and C. Grünewälder, "On the mechanism of biological transformation of citric acid. v. citrate synthase, a hydrolase for malyl coenzyme a.zum mechanismus der biologischen umwandlung von citronensaeure. v. citratsynthase, eine hydrolase fuer malyl-coenzym A," Biochemische Zeitschrift, vol. 339, pp. 436-453, 1964.

[2] P. Wunderwald and H. Eggerer, "18-O-studies with citrate synthase and malate synthase," European Journal of Biochemistry, vol. 11, no. 1, pp. 97-105, 1969.

[3] T. S. Raman and E. R. B. Shanmugasundaram, "The effects of two quaternary ammonium compounds on citric acid and sterol synthesis in Aspergillus niger," Journal of General Microbiology, vol. 31, pp. 23-29, 1963.

[4] S. Schlücker, B. Küstner, A. Punge, R. Bonfig, A. Marx, and P. Ströbel, "Immuno-Raman microspectroscopy: in situ detection of antigens in tissue specimens by surface-enhanced Raman scattering," Journal of Raman Spectroscopy, vol. 37, no. 7, pp. 719-721, 2006.

[5] J. De Gelder, K. De Gussem, P. Vandenabeele, P. De Vos, and L. Moens, "Methods for extracting biochemical information from bacterial Raman spectra: an explorative study on Cupriavidus metallidurans," Analytica Chimica Acta, vol. 585, no. 2, pp. 234-240, 2007.

[6] C. E. Nordman, A. S. Weldon, and A. L. Patterson, "X-ray crystal analysis of the substrates of aconitase-I. Rubidium dihydrogen citrate," Acta Crystallographica, vol. 13, pp. 414417, 1960.

[7] J. P. Glusker, J. A. Minkin, and A. L. Patterson, "X-ray crystal analysis of the substrates of aconitase-9. A refinement of the structure of anhydrous citric acid," Acta Crystallographica Section B, vol. 25, no. 6, pp. 1066-1072, 1969.

[8] D. E. Zacharias, J. P. Glusker, R. Guthrie, A. C. Sullivan, and P. A. Srere, "The structure of a citric anhydride derivative, $\mathrm{C}_{8} \mathrm{H}_{6} \mathrm{O}_{7}$," Acta Crystallographica Section C, vol. 40, pp. 2100 2103, 1984.

[9] R. Lemor, M. B. Kruger, D. M. Wieliczka, P. Spencer, and T. May, "Dentin etch chemistry investigated by Raman and infrared spectroscopy," Journal of Raman Spectroscopy, vol. 31, no. 3, pp. 171-176, 2000.

[10] J. L. Moreira and L. Santos, "Analysis of organic acids in wines by Fourier-transform infrared spectroscopy," Analytical and Bioanalytical Chemistry, vol. 382, no. 2, pp. 421-425, 2005.

[11] A. Nose, M. Myojin, M. Hojo, T. Ueda, and T. Okuda, "Proton nuclear magnetic resonance and Raman spectroscopic studies of Japanese sake, an alcoholic beverage," Journal of Bioscience and Bioengineering, vol. 99, no. 5, pp. 493-501, 2005.

[12] J. D. Tsay and T. T. Fang, "Effect of $\mathrm{pH}$ on the chemistry of the barium titanium citrate gel and its thermal decomposition behavior," Journal of the American Ceramic Society, vol. 84, no. 11, pp. 2475-2478, 2004.

[13] A. Caillet, N. Sheibat-Othman, and G. Fevotte, "Crystallization of monohydrate citric acid. 2. Modeling through population balance equations," Crystal Growth and Design, vol. 7, no. 10, pp. 2088-2095, 2007.

[14] Y. Xiong, J. M. McLellan, Y. Yin, and Y. Xia, "Synthesis of palladium icosahedra with twinned structure by blocking oxidative etching with citric acid or citrate ions," Angewandte Chemie, vol. 46, no. 5, pp. 790-794, 2007.

[15] P. Tarakeshwar and S. Manogaran, "Ground state vibrations of citric acid and the citrate trianion-an ab initio study," Spectrochimica Acta Part A, vol. 50, no. 14, pp. 2327-2343, 1994.
[16] P. K. Ghosh and D. S. Jayas, "Use of spectroscopic data for automation in food processing industry," in Proceedings of the American Society of Agricultural and Biological Engineers (ASABE '08), Providence, Ri, USA, June 2008.

[17] G. Rauhut and P. Pulay, "Transferable scaling factors for density functional derived vibrational force fields," Journal of Physical Chemistry, vol. 99, no. 10, pp. 3093-3100, 1995.

[18] G. Rauhut and P. Pulay, "Erratumml: transferable scaling factors for density functional derived vibrational force fields," Journal of Physical Chemistry, vol. 99, no. 39, Article ID 14572, 1995.

[19] F. Kalincsák and G. Pongor, "Extension of the density functional derived scaled quantum mechanical force field procedure," Spectrochimica Acta Part A, vol. 58, no. 5, pp. 9991011, 2002.

[20] S. F. Boys and F. Bernardi, "The calculation of small molecular interactions by the differences of separate total energies. Some procedures with reduced errors," Molecular Physics, vol. 19, no. 4, pp. 553-566, 1970.

[21] A. E. Reed, L. A. Curtiss, and F. Weinhold, "Intermolecular interactions from a natural bond orbital, donor-acceptor viewpoint," Chemical Reviews, vol. 88, no. 6, pp. 899-926, 1988.

[22] J. P. Foster and F. Weinhold, "Natural hybrid orbitals," Journal of the American Chemical Society, vol. 102, no. 24, pp. 72117218, 1980.

[23] A. E. Reed and F. Weinhold, "Natural localized molecular orbitals," The Journal of Chemical Physics, vol. 83, no. 4, pp. 1736-1740, 1985.

[24] E. D. Gledening, J. K. Badenhoop, A. D. Reed, J. E. Carpenter, and F. Weinhold, NBO 3.1, Theoretical Chemistry Institute, University of Wisconsin, Madison, Wis, USA, 1996.

[25] R. F. W. Bader, Atoms in Molecules, A Quantum Theory, Oxford University Press, Oxford, UK, 1990.

[26] F. B. Köning, J. Schönbohm, and D. Bayles, "AIM2000: a program to analyze and visualize atoms in molecules," Journal of Computational Chemistry, vol. 22, no. 5, pp. 545-559, 2001.

[27] A. D. Becke, "Density-functional thermochemistry. III. The role of exact exchange," The Journal of Chemical Physics, vol. 98, no. 7, pp. 5648-5652, 1993.

[28] C. Lee, W. Yang, and R. G. Parr, "Development of the ColleSalvetti correlation-energy formula into a functional of the electron density," Physical Reiew B, vol. 37, no. 2, pp. 785-789, 1988.

[29] M. J. Frisch, G. W. Trucks, H. B. Schlegel et al., Gaussian 03, Revision B.01, Gaussian inc., Pittsburgh, Pa, USA, 2003.

[30] T. Sundius, "Molvib-a flexible program for force field calculations," Journal of Molecular Structure, vol. 218, pp. 321326, 1990.

[31] T. Sundius, "Scaling of ab initio force fields by MOLVIB," Vibrational Spectroscopy, vol. 29, no. 1-2, pp. 89-95, 2002.

[32] G. Fogarasi, X. Zhou, P. W. Taylor, and P. Pulay, "The calculation of ab initio molecular geometries: efficient optimization by natural internal coordinates and empirical correction by offset forces," Journal of the American Chemical Society, vol. 114, no. 21, pp. 8191-8201, 1992.

[33] G. M. Florio, T. S. Zwier, E. M. Myshakin, K. D. Jordan, and E. L. Sibert, "Theoretical modeling of the $\mathrm{OH}$ stretch infrared spectrum of carboxylic acid dimers based on first-principles anharmonic couplings," Journal of Chemical Physics, vol. 118, no. 4, pp. 1735-1746, 2003.

[34] M. A. Palafox, J. L. Núñez, and M. Gil, “Theoretical quantum chemical study of benzoic acid: geometrical parameters and vibrational wavenumbers," International Journal of Quantum Chemistry, vol. 89, no. 1, pp. 1-24, 2002. 
[35] M. Plazanet, N. Fukushima, M. R. Johnson, A. J. Horsewill, and H. P. Trommsdorff, "The vibrational spectrum of crystalline benzoic acid: inelastic neutron scattering and density functional theory calculations," Journal of Chemical Physics, vol. 115, no. 7, pp. 3241-3248, 2001.

[36] S. A. Brandán, F. M. López, M. Montejo, J. J. López-González, and A. Ben Altabef, "Theoretical and experimental vibrational spectrum study of 4-hydroxybenzoic acid as monomer and dimer," Spectrochimica Acta-Part A, vol. 75, no. 5, pp. 14221434, 2010.

[37] P. Pulay, G. Fogarasi, F. Pang, and J. E. Boggs, "Systematic ab initio gradient calculation of molecular geometries, force constants, and dipole moment derivatives," Journal of the American Chemical Society, vol. 101, no. 10, pp. 2550-2560, 1979.

[38] P. Pulay, G. Fogarasi, G. Pongor, J. E. Boggs, and A. Vargha, "Combination of theoretical $\mathrm{ab}$ initio and experimental information to obtain reliable harmonic force constants. Scaled Quantum Mechanical (SQM) force fields for glyoxal, acrolein, butadiene, formaldehyde, and ethylene," Journal of the American Chemical Society, vol. 105, no. 24, pp. 7037-7047, 1983.

[39] A. B. Nielsen and A. J. Holder, Gauss View 3.0, User's Reference, Gaussian inc., Pittsburgh, Pa, USA, 2003.

[40] I. D. Reva and S. G. Stepanian, "An infrared study on matrixisolated benzoic acid," Journal of Molecular Spectroscopy and Molecular Structure, vol. 349, pp. 337-340, 1995.

[41] J. Zinczuk, A. E. Ledesma, S. A. Brandán, O. E. Piro, J. J. LópezGonzález, and A. Ben Altabef, "Structural and vibrational study of 2-(2'-furyl)-4,5-1H-dihydroimidazole," Journal of Physical Organic Chemistry, vol. 22, no. 12, pp. 1166-1177, 2009.

[42] A. E. Ledesma, S. A. Brandán, J. Zinczuk, O. E. Piro, J. J. López-González, and A. Ben Altabef, "Structural and vibrational study of 2-(2'-furyl)-1H-imidazole," Journal of Physical Organic Chemistry, vol. 21, no. 12, pp. 1086-1097, 2008. 


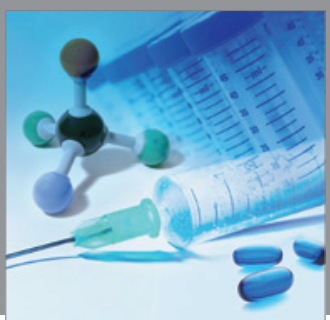

International Journal of

Medicinal Chemistry

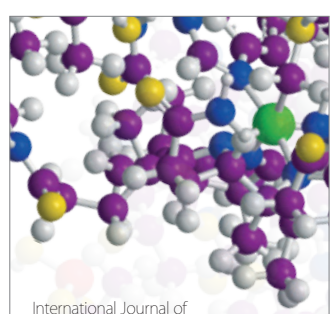

Carbohydrate Chemistry

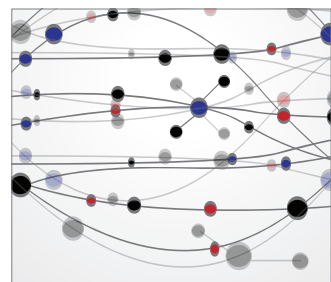

The Scientific World Journal
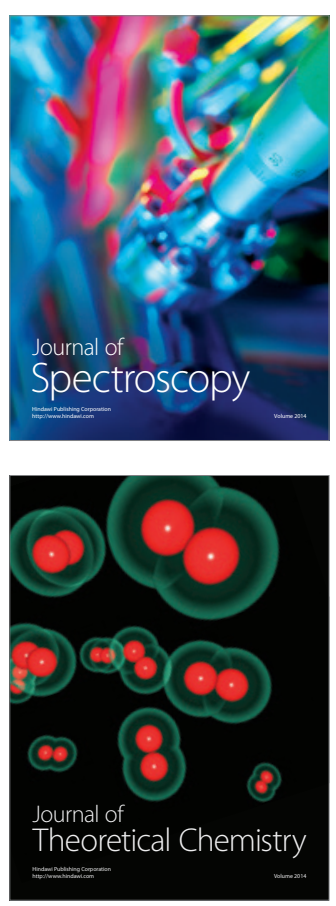
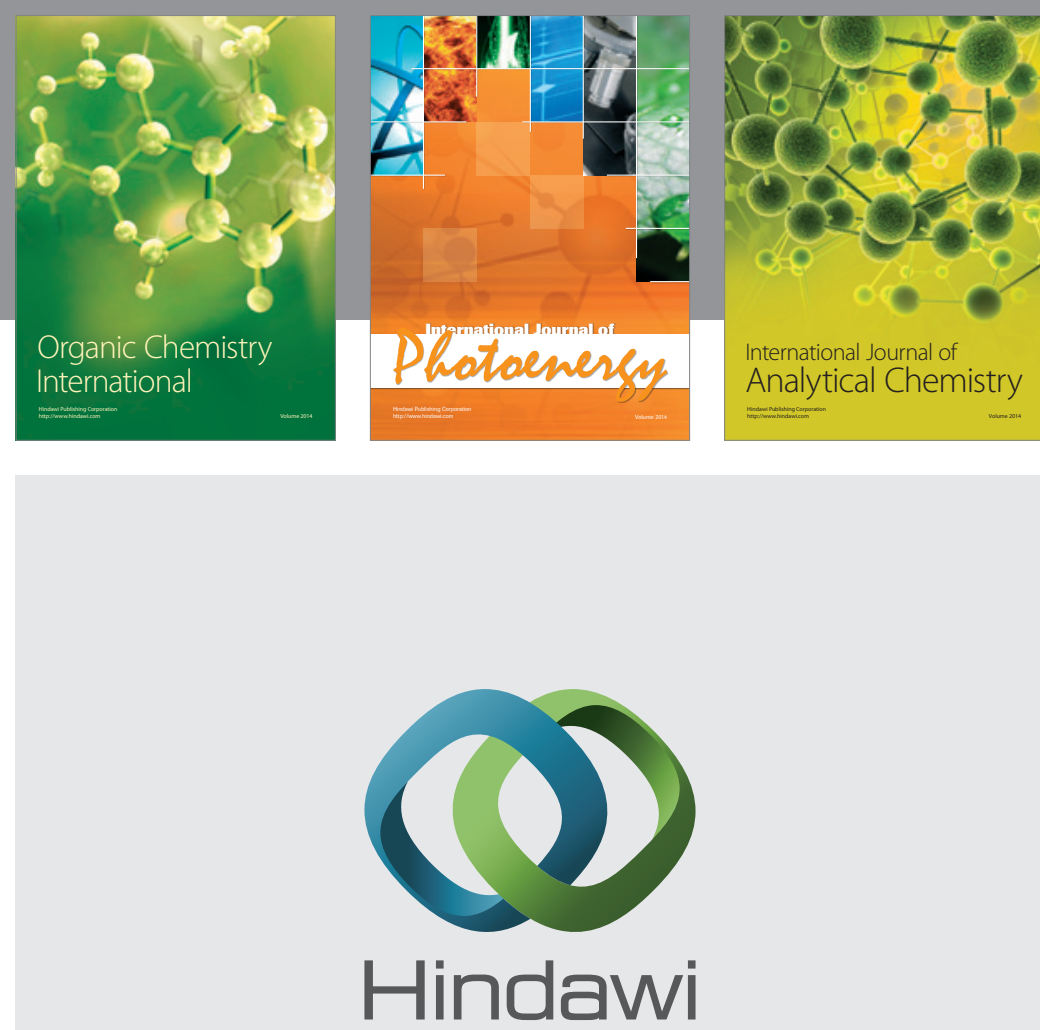

Submit your manuscripts at

http://www.hindawi.com
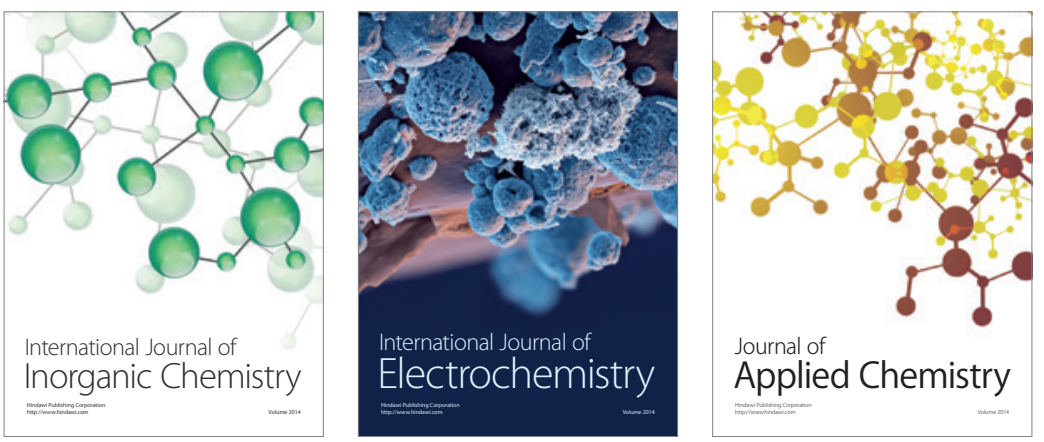

Journal of

Applied Chemistry
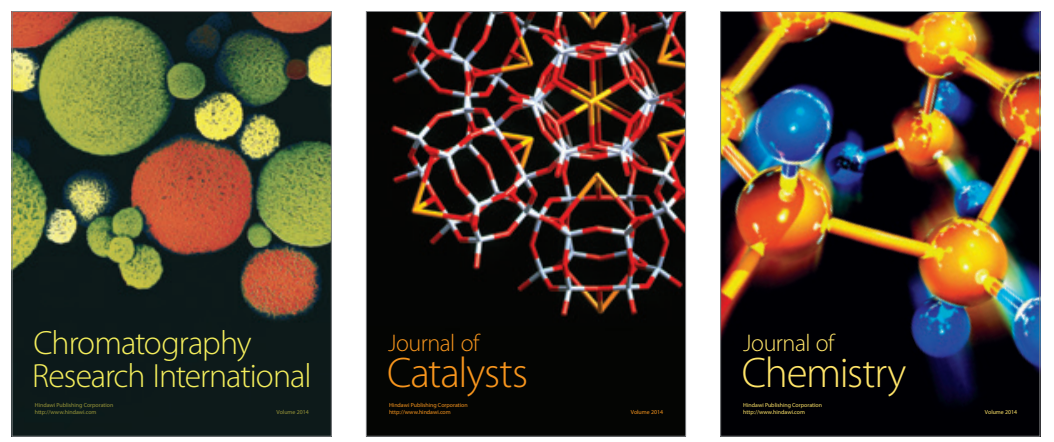
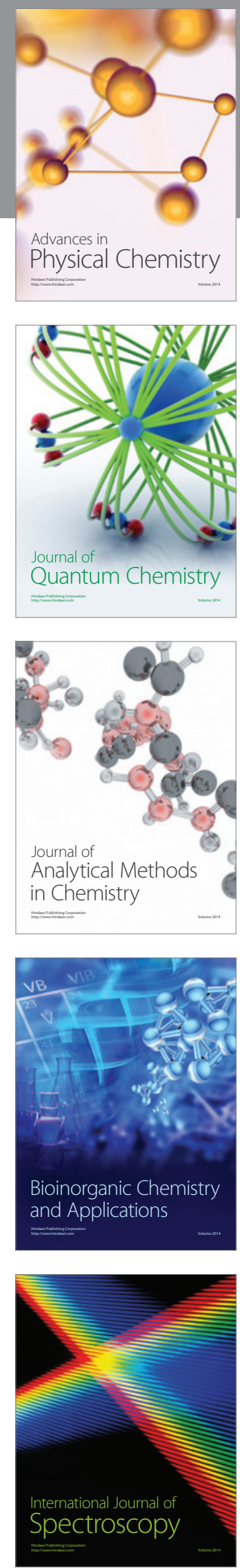